\title{
Coupling between a Charge Density Wave and Magnetism in an Heusler Material
}

\author{
G. Lantz, ${ }^{1}$ M. J. Neugebauer, ${ }^{1}$ M. Kubli, ${ }^{1}$ M. Savoini, ${ }^{1}$ E. Abreu, ${ }^{1}$ K. Tasca, ${ }^{1}$ C. Dornes,${ }^{1}$ \\ V. Esposito, ${ }^{2}$ J. Rittmann, ${ }^{2}$ Y. W. Windsor, ${ }^{2}$ P. Beaud, ${ }^{2}$ G. Ingold, ${ }^{2}$ and S. L. Johnson ${ }^{1}$ \\ ${ }^{1}$ Institute for Quantum Electronics, Physics Department, ETH Zurich, CH-8093 Zurich, Switzerland \\ ${ }^{2}$ Swiss Light Source, Paul Scherrer Institut, CH-5232 Villigen PSI, Switzerland \\ (Received 6 June 2017; revised manuscript received 27 September 2017; published 29 November 2017)
}

\begin{abstract}
The prototypical magnetic memory shape alloy $\mathrm{Ni}_{2} \mathrm{MnGa}$ undergoes various phase transitions as a function of the temperature, pressure, and doping. In the low-temperature phases below $260 \mathrm{~K}$, an incommensurate structural modulation occurs along the [110] direction which is thought to arise from the softening of a phonon mode. It is not at present clear how this phenomenon is related, if at all, to the magnetic memory effect. Here we report time-resolved measurements which track both the structural and magnetic components of the phase transition from the modulated cubic phase as it is brought into the highsymmetry phase. The results suggest that the photoinduced demagnetization modifies the Fermi surface in regions that couple strongly to the periodicity of the structural modulation through the nesting vector. The amplitude of the periodic lattice distortion, however, appears to be less affected by the demagnetization.
\end{abstract}

DOI: 10.1103/PhysRevLett.119.227207

The magnetic shape memory effect (MSME) in Heusler materials is characterized by a very large magnetic fieldinduced strain that can exceed $11 \%$ [1,2]. The particularly large MSME seen in Ni-Mn-Ga alloys is promising for technological applications [2-4]. Stoichiometric $\mathrm{Ni}_{2} \mathrm{MnGa}$ exhibits MSME only in its lowest temperature phase. At higher temperatures, $\mathrm{Ni}_{2} \mathrm{MnGa}$ undergoes a series of structural and magnetic phase transitions. The lowtemperature phase, characterized by a martensitic tetragonal structure (MT) with an incommensurate structural modulation, is stable up to $T_{\mathrm{MT}}=220 \mathrm{~K}$. Between 220 and $260 \mathrm{~K}$ lies the so-called premartensitic (PMT) phase, which can be described as a cubic austenitic phase with an incommensurate structural modulation. Between 260 and $380 \mathrm{~K}$, this modulation disappears and the material is in the austenitic cubic phase (AUS). All the phases below the Curie temperature of $380 \mathrm{~K}$ are ferromagnetic.

The MSME is widely understood to originate from the reorientation of twinned domains in the tetragonal phase. The microscopic mechanism of the structural modulation and its link to both the MSME and ferromagnetism are, however, not completely understood. Since the modulation, upon cooling, arises already in the PMT phase, understanding its origin in this phase might shed light on its potential link to the MSME. Studying the modulation in the PMT phase presents the advantage of maintaining the same lattice parameters during the PMT-AUS phase transition, which allows sensitivity to only the modulation dynamics. The modulation is incommensurate and is thought to be triggered by a phonon softening along the [110] direction $[5,6]$. The modulation can be understood within two scenarios. The first is an adaptive model, where the modulation is considered as an effective twinning arising from minimization of elastic energy [7,8]. The other scenario is Fermi surface nesting, which results in a phonon softening and the appearance of a charge density wave (CDW). The latter has recently gotten more experimental and theoretical support [9-13]. A unified theory has been proposed by Gruner et al., in which the adaptive nanotwinning is locked on the CDW [14]. Schubert et al. [15] have shown that doping with magnetic cobalt impurities causes a change in both the spatial periodicity of the modulation and the associated amplitude mode associated with the modulation. While this is indicative of a possible link between magnetism and the CDW, Ni-Mn-Ga alloys are very sensitive to chemical doping, and the changes observed could also be caused by local structural or electronic modifications [16].

Time-resolved pump-probe experiments offer the opportunity to study the coupling of magnetism and structure in materials following a sudden perturbation. Previous pumpprobe experiments on $\mathrm{Ni}_{2} \mathrm{MnGa}$ have tracked the amplitude mode of the CDW across the MT-AUS phase transition $[15,17,18]$. In this Letter, we study the dynamics of stoichiometric $\mathrm{Ni}_{2} \mathrm{MnGa}$ starting from the PMT phase using the time-resolved magnetic optical Kerr effect (MOKE) and time-resolved $\mathrm{x}$-ray diffraction (trXRD). This combination of techniques enables us to follow separately the structural modulation and the magnetism after strong electronic excitation from a femtosecond pulse of light.

The same sample was used for all our measurements, a single crystal of stoichiometric $\mathrm{Ni}_{2} \mathrm{MnGa}$ from AdaptaMat Ltd. [001]-cut and has a $5 M$ structure in the MT phase. The PMT and MT transition temperatures were measured at $T_{\mathrm{PMT}}=264 \mathrm{~K}$ and $T_{\mathrm{MT}}=224 \mathrm{~K}$, respectively [17].

The MOKE experiment was performed in a polar configuration with a nearly collinear pump probe along 
the surface normal in a $0.7 \mathrm{~T}$ field, sufficient to saturate the magnetization (see Supplemental Material [19]). A $250 \mathrm{kHz}$ Ti-sapphire laser system was used to deliver 45-fslong $800 \mathrm{~nm}$ pump pulses and $400 \mathrm{~nm}$ frequency-doubled probe pulses. Probe and pump attenuation lengths were 16 and $23 \mathrm{~nm}$, respectively. We estimate the transient magnetization of the sample by measuring the Kerr rotation, which is the real part of the full complex Kerr angle (see Supplemental Material [19]) [20,21]. In both experiments, the sample temperature was set to $225 \mathrm{~K}$, well within the PMT phase, using a nitrogen cryogenic blower.

The trXRD experiment was performed at the FEMTO slicing source of the Swiss Light Source (PSI, Villigen, Switzerland) [22]. The $7 \mathrm{keV} \mathrm{x}$-ray beam was set at grazing incidence $\left(0.65^{\circ}\right)$, resulting in an attenuation length of $55 \mathrm{~nm}$. The $800 \mathrm{~nm}$ pump pulse had an incidence angle of $12^{\circ}$, resulting in an attenuation length depth of $23 \mathrm{~nm}$ [23]. The penetration depth mismatch between the pump and probe leads to a smearing of the observed dynamics that has to be accounted for in the modeling. The overall time resolution for these measurements was better than $150 \mathrm{fs}$ [22] (see Supplemental Material [19]). The sample was previously oriented at the Material Science beam line of the Swiss Light Source [24], where the orientation matrix was computed and then verified during the time-resolved experiment. An avalanche photodiode was used to measure the $\mathrm{x}$-ray intensity for each pulse.

Figure 1(a) shows the magnetization dynamics for several fluences. An initial drop over a time scale of $1 \mathrm{ps}$ is followed by a second time scale of about $10 \mathrm{ps,} \mathrm{over} \mathrm{which} \mathrm{the}$ magnetization either partially recovers by a small amount (at low fluences) or further decreases (at high fluences). The fluence dependence of the relative change in magnetization is shown in Fig. 1(b) at both 1 and 20 ps after excitation, along with a linear fit. The changes at $1 \mathrm{ps}$ agree well with this linear model, whereas the later time changes do not.

For comparison, Fig. 1(c) shows the time-dependent changes in the $x$-ray intensity at the maximum of the rocking curve of the $(2-q 02-q)$ superlattice reflection, where $q=0.341$ [6], over a similar range of excitation fluences as in Fig. 1(a). These dynamics are more complex but reveal a fast decrease in intensity over the first few picoseconds followed by slower dynamics which are somewhat reminiscent of the behavior of the magnetization. At late times, we see a fluence-dependent partial recovery of the superlattice intensity over a time scale of 4-12 ps, which at the highest fluences is followed by a further decrease in intensity. Because of the penetration depth mismatch between the probe and pump beams, the trXRD measurement is an average over regions with different electronic excitation densities. In order to quantitatively compare MOKE and trXRD measurements, we convert the incident fluence into the average excitation density $\left\langle n_{0}\right\rangle$ seen by the probe (see Supplemental Material [19]). Figure 1(d) shows the normalized difference in measured
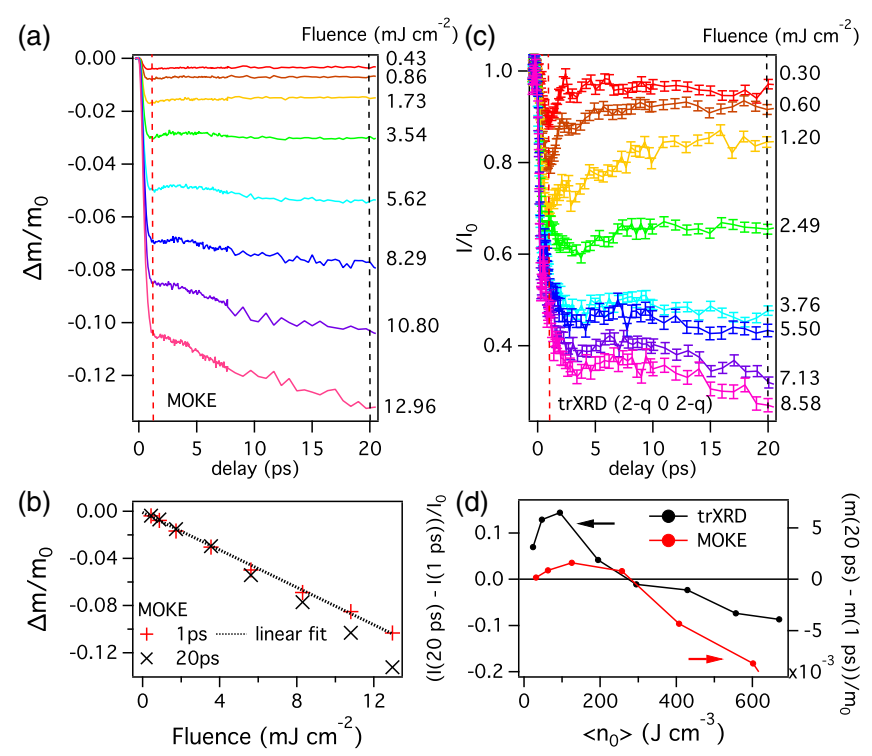

FIG. 1. Comparison between MOKE and trXRD. (a) Relative changes in the MOKE signal for difference fluences. The red and black dotted lines show the 1 and 20 ps delays, respectively. (b) Difference in magnetization at 1 and 20 ps versus fluence. The dotted line shows the linear behavior versus fluence of the demagnetization at 1 ps delay and the nonlinearity of the $20 \mathrm{ps}$ delay. (c) $(2-q \quad 0 \quad 2-q)$ intensity for different fluences. (d) Difference in demagnetization and loss of intensity for MOKE and trXRD between 20 and 1 ps delays versus observed excitation density $\left\langle n_{0}\right\rangle$. The difference is positive up to $300 \mathrm{~J} \mathrm{~cm}^{-3}$ and becomes negative after for both MOKE and trXRD.

intensities at $20 \mathrm{ps}$ and at $1 \mathrm{ps}$ for both MOKE and trXRD, as a function of $\left\langle n_{0}\right\rangle$, yielding to a similar behavior: At excitation densities below $300 \mathrm{~J} \mathrm{~cm}^{-3}$, both the magnetization and diffracted intensity partially recover, whereas above this value they both further decrease over this time scale. Regarding the longer time dynamics shown in Fig. 1(c), we tentatively attribute the partial recovery at intermediate time scales as a consequence of the cooling of the excited electrons to the lattice. The somewhat longer time scale decrease seen at higher fluences may result from a more conventional temperature-driven phase transition as the lattice heats and internally equilibrates to a temperature past the transition temperature. Complementary measurements of the electronic and lattice temperature by timeresolved angle-resolved photoemission spectroscopy and time-resolved diffuse scattering would be some possible ways to further investigate these processes [25-27].

In order to study possible time-dependent changes in the modulation wave vector, we show in Fig. 2 the diffracted intensity as a function of rotation angle $\varphi$ about the surface normal for different time delays for both the (202) and its satellite reflection $(2-q 02-q)$. A fit using a Voigt function was performed to extract the precise peak position, $\varphi_{p}$. Figure 2(c) shows the resulting $\varphi_{p}$ values. We first consider the (202) Bragg reflection. $\varphi_{p}$ of the (202) 

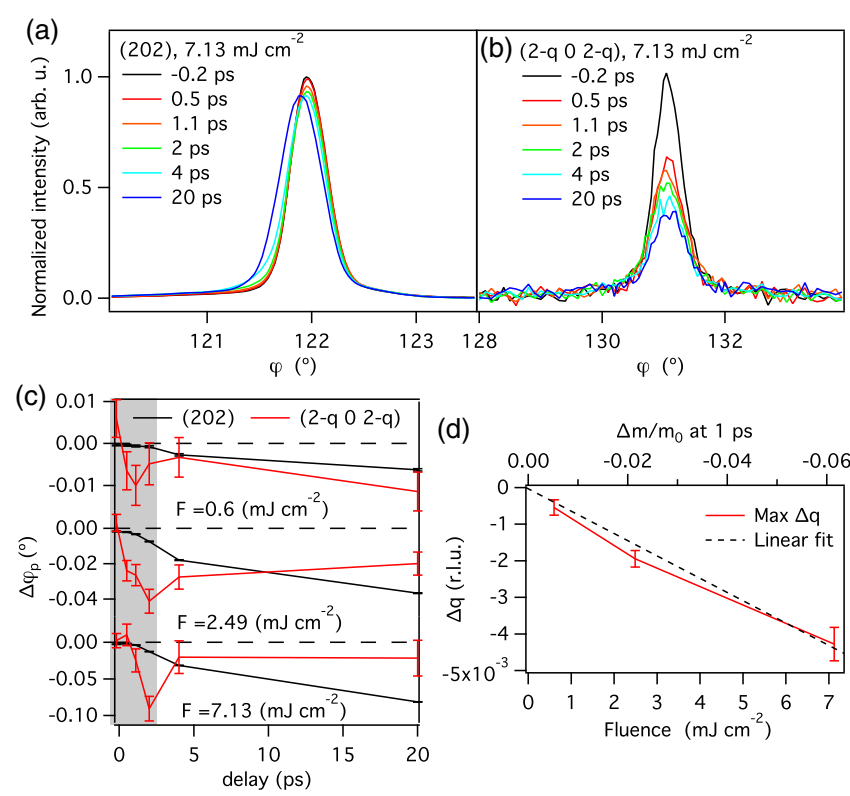

FIG. 2. Rotation scans at a $7.13 \mathrm{~mJ} \mathrm{~cm}^{-2}$ fluence for the (a) (202) Bragg reflection and (b) $(2-q 02-q)$ superlattice reflection. (c) Peak positions versus time for different fluences. The (202) does not shift significantly before $4 \mathrm{ps,} \mathrm{whereas} \mathrm{the}$ $(2-q 02-q)$ reaches its minimum around $2 \mathrm{ps}$. The gray region represents the times for which the orientation matrix is assumed to be nearly unchanged in order to estimate $\Delta q$ of the superlattice distortion. (d) Extracted maximum $\Delta q$ change versus fluence (bottom axis) and demagnetization value at 1 ps from Fig. 1(c) (top axis).

reflection decreases monotonically, consistent with an uniaxial expansion of the crystal along the surface normal which is typical for laser-driven heating [28]. This expansion takes place over a time scale determined by strain wave propagation from the surface of the crystal through the probed region, estimated as $\delta / v=12.5 \mathrm{ps,} \mathrm{with} \mathrm{a} \mathrm{probe}$ depth $\delta=55 \mathrm{~nm}$ and a speed of sound $v=4.4 \mathrm{~nm} \mathrm{ps}^{-1}$ [29]. Over the first two picoseconds, the value of $\varphi_{p}$ shifts less than $0.02^{\circ}$ even at the highest fluence. At 20 ps delay, the strain can be estimated at $0.6 \%$ for the maximum fluence of $7.13 \mathrm{~mJ} \mathrm{~cm}^{-2}$ [28]. The time scale of the expansion matches the tens of picoseconds time scale seen in the magnetization and satellite reflection dynamics (Fig. 1). At high fluences, this is consistent with equilibrium measurements that show that both the modulation and the magnetization decrease with increasing strain along the [001] direction [30,31], although at low fluences we see that these are anticorrelated in the time-resolved measurements, which makes it difficult to draw a definite conclusion about the relationship between strain, magnetization, and the modulation amplitude in the nonequilibrium case.

In marked contrast to the dynamics of $\varphi_{p}$ for the (202) reflection, $\varphi_{p}$ for the satellite reflection shows larger changes that are maximal at 1-2 ps after excitation, after which $\varphi_{p}$ relaxes back toward its initial unexcited value.
This suggests that $q$ changes independently of the dynamics of the underlying unit cell dimensions. Under the rough assumption that over the first few picoseconds these unit cell dimensions remain at their initial, unexcited values and that the direction of the modulation vector is unchanged, we can relate the measured changes in $\varphi_{p}$ to changes in the magnitude of the superlattice modulation wave vector $q$. Figure 2(d) shows $\Delta q$, the maximum value of the timedependent change in $q$, as a function of the fluence. $\Delta q$ is approximately proportional to the fluence, as evidenced by the dashed linear fit to the data. Note also that $\Delta q$ decreases with increasing fluence, contrary to what happens upon increasing the temperature [12]. The fact that $\varphi_{p}(t)$ is qualitatively different from the time dependence of the intensity provides some confidence that the apparent small changes are indeed reflective of a genuine change in the modulation wave vector.

Both the rapid onset of these changes in $q$ as well as their linearity with respect to the fluence are quite similar to the initial behavior of the magnetization dynamics [Fig. 1(b)]. The magnetization drops on a time scale of $1 \mathrm{ps}$, which is slightly faster than the 2 ps decrease seen in $q$ for most fluences. On this basis, we suggest that the demagnetization drives the $q$ shift. In equilibrium, Lee, Rhee, and Harmon [32] have shown that changes in the magnetization density lead to proportional changes in q. In Fig. 2(d), we show on the top axis the experimental demagnetization value at 1 ps from Fig. 1(b) for an equivalent fluence. Using the theoretical $q$ shift versus magnetization from Ref. [32] and assuming that the sample is initially at $80 \%$ magnetization saturation, we calculate that a fluence of $7.13 \mathrm{~mJ} \mathrm{~cm}^{-2}$ leads to $\Delta q=-0.016,4$ times lower than the measured trXRD value. Despite this quantitative disagreement, the signs of shifts and their linearity are consistent with the idea of the demagnetization playing some role. The quantitative discrepancy may be related to additional contributions to the Fermi surface nesting arising from the highly excited electronic states. The decrease of $q$ with demagnetization closely resembles the increase of the real space periodicity in the phase transitions from $5 M$ to $7 M$ to nonmodulated structures that occur during compression stress [2].

We now turn our attention to the ten first picoseconds of the satellite reflection intensity, which tracks the magnitude of the structural modulation, summarized in Fig. 3(a), an enlarged version of Fig. 1(c). First, we note that the dynamics are markedly nonlinear with respect to the absorbed fluence. At fluences up to $1.2 \mathrm{~mJ} \mathrm{~cm}^{-2}$, the changes appear largely consistent with a displaced coherent excitation model [33] of the amplitude mode of the CDW, where the displaced mode has a frequency $\nu \cong 0.35 \mathrm{THz}$. As the fluence increases beyond $2 \mathrm{~mJ} \mathrm{~cm}^{-2}$, however, the time dependence changes dramatically. Most notably, the frequency of oscillation increases by up to a factor of 4 relative to the low-fluence behavior. This increase in the frequency with fluence stands in contrast to the temperature 


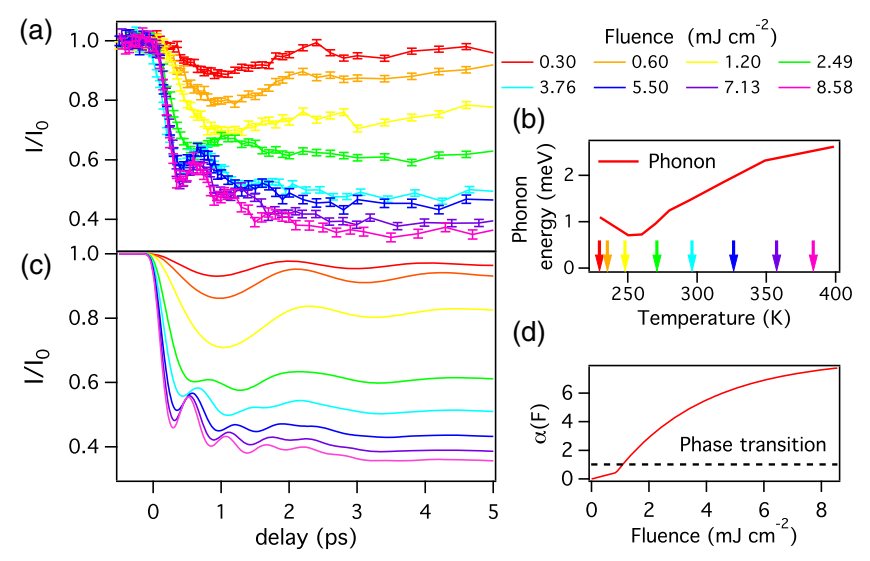

FIG. 3. trXRD intensity compared to single parameter model. (a) trXRD time scan of the superlattice reflection for several fluences. (b) Phonon energy versus temperature, showing the softening (adapted from Ref. [5]). The colored arrows show the average estimated final temperature reached by the excited area at different fluences. (c) Fitted single parameter model. (d) Fluence dependence of the excitation function $\alpha(F)$.

dependence of the amplitude mode frequency, where an increase in the temperature causes a decrease in the frequency [15]. An increase in the frequency in the coherent oscillations of a superlattice reflection has been seen in other structurally modulated materials [34,35]; in those two cases, a doubling of the frequency is reported, which was attributed to a change in the symmetry of the interatomic potential consistent with a phase transition to a higher symmetry state. In our case, we see a much more dramatic increase in the frequency, which can, however, be modeled in a similar way.

We consider that locally the magnitude of the structural modulation can be represented by a single variable $\eta$. To treat the dynamics of the phase transition, we consider an effective potential energy which has the same functional dependence on $\eta$ as does the Landau free energy. This should, in principle, correspond to the phase transition order, which is first order for the PMT to AUS transition in equilibrium conditions. However, the measured hysteresis and latent heat of the transition are very small [12]. Therefore, we can consider that the transition is close to second order [31,36] (see Supplemental Material [19]). We assume that the contribution of $\eta$ to the potential energy is of the form

$$
V(\eta)=\frac{1}{2}[\alpha(F)-1] \eta^{2}+\frac{1}{4} \eta^{4},
$$

where $\alpha(F)$ is a function of the fluence $F$. The shape of the potential has its minimum at $\eta=1$ when $\alpha(0)=0$ in order to use a normalized intensity. The time dependence is computed using the following equation of motion:

$$
\frac{1}{\omega_{\mathrm{DW}}^{2}} \frac{\partial^{2}}{\partial^{2} t} \eta+\frac{\partial}{\partial \eta} V(\eta)+\frac{\gamma}{\omega_{\mathrm{DW}}^{2}} \frac{\partial}{\partial t} \eta=0
$$

where $\omega_{\mathrm{DW}}$ is the frequency in the double well potential at $\alpha=0$ and $\gamma$ is the damping rate. The diffracted $\mathrm{x}$-ray intensity $I$ is then proportional to $\eta^{2}$. Initially, $\alpha(0)=0$, which is changed instantaneously to $\alpha(F)$ at $t_{0}$, the arrival time of the pump pulse. This sudden change causes the system to oscillate in the new potential, which is fully determined by the function $\alpha(F)$.

In Refs. [34,35], the excitation function $(\alpha)$ is assumed to be proportional to $F$, in close analogy to the temperature dependence in the Landau theory $[31,37,38]$. Some modifications of this model are needed to account for the fourfold increase in the frequency observed in the present case. As a guide to the functional form of $\alpha(F)$, we consider the experimentally measured temperature dependence of the phonon frequency associated with the amplitude mode, shown in Fig. 3(b). We have marked the temperatures corresponding to the excitation fluences used, under the assumption that the energy density deposited by the laser fully equilibrates with the lattice and that transport effects are negligible [39] (Supplemental Material [19]). The lowest three temperatures or fluences are below the phase transition, which could explain the sudden change in the dynamics above $2 \mathrm{~mJ} \mathrm{~cm}^{-2}$. Using the phonon energy versus temperature as a guide, we construct $\alpha(F)$, linear up to a critical fluence and exponential above:

$$
\begin{aligned}
& \alpha(F)=a F \quad \text { for } F<F_{c}, \\
& \alpha(F)=a F_{c}+b\left(1-e^{-\left(F-F_{c}\right) / d}\right) \quad \text { for } F>F_{c},
\end{aligned}
$$

where $a, b, d$, and $F_{c}$ are constants. We perform a global fit of all curves simultaneously, using $a, b, d, F_{c}, \omega_{\mathrm{DW}}$, and $\gamma$ as fitting parameters. We fit the first $10 \mathrm{ps}$ and ignore the strain contribution. Since the intensity of the laser decays exponentially inside the material, the absorbed energy has a strong depth dependence, which we account for by dividing the material into layers of thickness $\delta=1 \mathrm{~nm}$ with different absorbed energy. Each layer has a new potential, determined by $\alpha(F)$, which is then used in the equation of motion. Since the correlation length of the material is small, different layers contribute incoherently to the signal such that the global intensity is the sum over all the layers weighed by the $\mathrm{x}$-ray penetration depth.

The best fit result is shown in Fig. 3(c) using $\alpha(F)$ shown in Fig. 3(d). The dynamics are well reproduced. It is worth noticing that $\alpha\left(F_{c}\right)<1$, which is lower than the excitation parameter that triggers the phase transition. Similarly, the soft phonon at equilibrium does not reach zero at $T_{\mathrm{PMT}}$ but by interpolation would reach zero at $T_{\mathrm{PMT}}-5 \mathrm{~K}$. The excitation parameter $\alpha(F)$ is nonlinear and follows a similar behavior as the phonon softening, showing that the amplitude of the modulation is mostly uncorrelated to the magnetism. The latter is linear versus the fluence and not needed to model the amplitude dynamics.

In conclusion, we have separately probed the magnetization and the structural modulation using MOKE and 
trXRD, respectively, starting from the PMT phase, and analyzed the correlation between the two. We show that strain propagation contributes to shifting the Bragg reflection and lowering the magnetization on a time scale of tens of picoseconds. On a shorter time scale, the superlattice peak shifts linearly with the fluence. We suggest that the photoinduced demagnetization modifies the Fermi surface, which in turn changes the magnitude of the nesting vector and therefore the modulation periodicity. In contrast, the amplitude of the modulation has a nonlinear fluence behavior which can be modeled with a single variable, $\eta$, potential energy fully determined by an excitation parameter. The fluence dependence of this parameter is similar to the behavior of the phonon softening with the temperature. It thus appears that the magnetization has some impact on the periodicity of the lattice distortion but does not strongly determine its amplitude. The suggested correlation between magnetism and modulation periodicity may be crucial for understanding the precise mechanism of the MSME.

Time-resolved $\mathrm{x}$-ray diffraction measurements were carried out at the X05LA beam line, and preparative static grazing incidence diffraction measurements were performed at the X04SA beam line of the Swiss Light Source, Paul Scherrer Institut, Villigen. We acknowledge financial support by the NCCR Molecular Ultrafast Science and Technology (NCCR MUST), a research instrument of the Swiss National Science Foundation (SNSF). G. L. also acknowledges the financial support of ETH Career Seed Grant No. SEED-80 16-1. E. A. acknowledges support from the ETH Zurich Postdoctoral Fellowship Program and from the Marie Curie Actions for People COFUND Program.

[1] A. Sozinov, A. A. Likhachev, N. Lanska, and K. Ullakko, Appl. Phys. Lett. 80, 1746 (2002).

[2] E. Pagounis, M. J. Szczerba, R. Chulist, and M. Laufenberg, Appl. Phys. Lett. 107, 152407 (2015).

[3] N. Gabdullin and S. H. Khan, IEEE Trans. Magn. 52, 1 (2016).

[4] A. Sozinov, N. Lanska, A. Soroka, and W. Zou, Appl. Phys. Lett. 102, 021902 (2013).

[5] A. Zheludev, S. M. Shapiro, P. Wochner, A. Schwartz, M. Wall, and L. E. Tanner, Phys. Rev. B 51, 11310 (1995).

[6] S. Mariager, T. Huber, and G. Ingold, Acta Mater. 66, 192 (2014).

[7] A. G. Khachaturyan, S. M. Shapiro, and S. Semenovskaya, Phys. Rev. B 43, 10832 (1991).

[8] S. Kaufmann, U. K. Rößler, O. Heczko, M. Wuttig, J. Buschbeck, L. Schultz, and S. Fähler, Phys. Rev. Lett. 104, 145702 (2010)

[9] C. Bungaro, K. M. Rabe, and A. Dal Corso, Phys. Rev. B 68, 134104 (2003).

[10] S. M. Shapiro, P. Vorderwisch, K. Habicht, K. Hradil, and H. Schneider, Europhys. Lett. 77, 56004 (2007).
[11] S. W. D’Souza, A. Rai, J. Nayak, M. Maniraj, R. S. Dhaka, S. R. Barman, D. L. Schlagel, T. A. Lograsso, and A. Chakrabarti, Phys. Rev. B 85, 085123 (2012).

[12] S. Singh, J. Bednarcik, S. R. Barman, C. Felser, and D. Pandey, Phys. Rev. B 92, 054112 (2015).

[13] M. Belesi, L. Giebeler, C. G. F. Blum, U. K. Rößler, B. Büchner, and S. Wurmehl, Phys. Rev. B 91, 134415 (2015).

[14] M. E. Gruner, R. Niemann, P. Entel, R. Pentcheva, U. K. Rössler, K. Nielsch, and S. Fähler, arXiv:1701.01562.

[15] M. Schubert et al., Phys. Rev. Lett. 115, 076402 (2015).

[16] A. Planes, L. Mañosa, and M. Acet, J. Phys. Condens. Matter 21, 233201 (2009).

[17] S. O. Mariager, A. Caviezel, P. Beaud, C. Quitmann, and G. Ingold, Appl. Phys. Lett. 100, 261911 (2012).

[18] S. O. Mariager et al., Phys. Rev. B 90, 161103 (2014).

[19] See Supplemental Material at http://link.aps.org/ supplemental/10.1103/PhysRevLett.119.227207 for excitation density calculations, static magnetic measurements, temperature calculations, and expanding the energy potential to the sixth order.

[20] I. Razdolski, A. Alekhin, U. Martens, D. Bürstel, D. Diesing, M. Münzenberg, U. Bovensiepen, and A. Melnikov, J. Phys. Condens. Matter 29, 174002 (2017).

[21] E. Carpene, F. Boschini, H. Hedayat, C. Piovera, C. Dallera, E. Puppin, M. Mansurova, M. Münzenberg, X. Zhang, and A. Gupta, Phys. Rev. B 87, 174437 (2013).

[22] P. Beaud, S. L. Johnson, A. Streun, R. Abela, D. Abramsohn, D. Grolimund, F. Krasniqi, T. Schmidt, V. Schlott, and G. Ingold, Phys. Rev. Lett. 99, 174801 (2007).

[23] Y. V. Kudryavtsev, Y. P. Lee, and J. Y. Rhee, Phys. Rev. B 66, 115114 (2002).

[24] P. R. Willmott et al., J. Synchrotron Radiat. 20, 667 (2013).

[25] M. Trigo et al., Nat. Phys. 9, 790 (2013).

[26] G. Lantz et al., Nat. Commun. 8, 13917 (2017).

[27] T. D. Haynes, R. J. Watts, J Laverock, Z. S. Major, M. A. Alam, J. W. Taylor, J. A. Duffy, and S. B. Dugdale, New J. Phys. 14, 035020 (2012).

[28] S. L. Johnson, P. Beaud, E. Vorobeva, C. J. Milne, É. D. Murray, S. Fahy, and G. Ingold, Acta Crystallogr. Sect. A 66, 157 (2010).

[29] T. E. Stenger and J. Trivisonno, Phys. Rev. B 57, 2735 (1998).

[30] Z. Nie, Y. Wang, S. Shang, Q. Zeng, Y. Ren, D. Liu, W. Yang, Y. Wang, and Z.-K. Liu, Appl. Phys. Lett. 106, 021910 (2015).

[31] A. Planes, E. Obradó, A. Gonzàlez-Comas, and L. Mañosa, Phys. Rev. Lett. 79, 3926 (1997).

[32] Y. Lee, J. Y. Rhee, and B. N. Harmon, Phys. Rev. B 66, 054424 (2002).

[33] H. J. Zeiger, J. Vidal, T. K. Cheng, E. P. Ippen, G. Dresselhaus, and M. S. Dresselhaus, Phys. Rev. B 45, 768 (1992).

[34] T. Huber et al., Phys. Rev. Lett. 113, 026401 (2014).

[35] P. Beaud et al., Nat. Mater. 13, 923 (2014).

[36] O. Velikokhatnyi and I. Naumov, Phys. Solid State 41, 617 (1999).

[37] V. Buchelnikov, A. Zayak, A. Vasil'ev, and T. Takagi, Int. J. Appl. Electromagnet. Mech. 12, 19 (2000).

[38] R. J. Gooding and J. A. Krumhansl, Phys. Rev. B 39, 1535 (1989).

[39] M. A. Uijttewaal, T. Hickel, J. Neugebauer, M. E. Gruner, and P. Entel, Phys. Rev. Lett. 102, 035702 (2009). 